\section{Design and Production of Two-piece Thyroid-neck Phantom by the Concurrent Use of Epoxy Resin and Poly(methyl methacrylate) Soft Tissue Equivalent Materials}

Karimi M. ${ }^{1}$, Mostaghimi H. ${ }^{1,2 *}$, Shams S. F. ${ }^{1}$, Mehdizadeh A. R. ${ }^{1}$

\begin{abstract}
The aim of this report is to present a new two-piece thyroid-neck phantom produced by the concurrent use of epoxy resin and poly(methyl methacrylate) (PMMA: plexiglass) soft tissue equivalent materials. Accordingly, mass attenuation coefficients of the epoxy resin and the plexiglass compounds were obtained from simulation (NIST XCOM 3.1) and measurements (practical dosimetry) and compared to those related to human soft tissue (ICRU 44). The thyroid-neck phantom and thyroid gland dimensions were derived from scientific references and the atlas of human anatomy, respectively. The thyroid phantom was designed by CATIA V5R16 software and produced by the epoxy resin compound by three-dimensional printer. Other organs were designed by ProNest software and made by the plexiglass sheets by $\mathrm{CNC}$ laser cutting machine. The mass attenuation coefficients for the epoxy resin $(50 \mathrm{keV}-20 \mathrm{MeV})$ and the plexiglass $(0-$ $20 \mathrm{MeV}$ ) were comparable to human soft tissue (ICRU 44), all with standard relative deviation beneath 5\%. In addition, the SPECT images indicated the similarity between human thyroid tissue and its phantom. In conclusion, this study proves the feasibility and reliability of epoxy resin application in the production of two-piece thyroid-neck phantom. This phantom can be applied in the calibration of gamma camera systems, dosimetry and gamma spectrometry in the nuclear medicine field.
\end{abstract}

Keywords

Thyroid-neck Phantom, Nuclear Medicine, Epoxy Resin, Plexiglass

\section{Introduction}

$\tau$ $\mathrm{n}$ the therapeutic and diagnostic processes of nuclear medicine, a radiopharmaceutical is administered to the patient orally or intravenously which exposes the patients, occupational staff and general public to radiation exposure. Currently, there are many nuclear medical centers around the world, which mainly use ionizing radiation in diagnosis or treatment. In most centers, working personnel perform different tasks such as avoidance, fractionation, labelling and injection, which increase the risk of exposure and require the implementation of regular instructions and monitoring to compute the internal dosages of staff [ 1 , 2]. In particular, it is necessary to evaluate the risk of the dose prescribed by therapeutic methods for the patient. Therefore, a standard method is necessary to estimate the internal doses in patients $[3,4]$.

The evaluation of internal doses of radionuclide depends both on the total number of nuclear transformations of radionuclide around the
${ }^{1}$ Department of Biomedical Physics and

Engineering, School of

Medicine, Shiraz Univer-

sity of Medical Sciences,

Shiraz, Iran

${ }^{2}$ Advanced Health

Technologies Research

Center, Shiraz University

of Medical Sciences,

Shiraz, Iran

*Corresponding author:

H. Mostaghimi

Department of Bio-

medical Physics and

Engineering, School of

Medicine, Shiraz Univer-

sity of Medical Sciences,

Shiraz, Iran

E-mail: mostaghimi@

sums.ac.ir

Received: 20 April 2018 Accepted: 24 May 2018 
source and on the energy absorbed per unit mass in the target tissue $[5,6]$. The radioactivity of such sources can be measured using quantitative imaging, direct measurement in the whole body or specific organs, or by indirect measurement in biological specimens especially in urine. The absorbed dose in the target tissue can also be calculated by Monte Carlo simulations [5-7] or Kernel Point doses [8].

In order to measure the absorbed dose, anthropomorphic phantoms are used [5]. These phantoms are made from materials with an attenuation coefficient similar to that of human tissue and also represent the anatomical shape and geometrical dimensions of the target tissue. Phantoms are also used for quality control, calibration and correction of imaging devices such as gamma camera systems. Current commercially-available thyroid-neck phantoms are fabricated with poly(methyl methacrylate)(PMMA: Plexiglass).

In this research, we aim to construct a phantom by the concurrent use of plexiglass and epoxy resin materials for the first time. Since epoxy resin is not used in the designation of these phantoms, the study of physical properties such as mass density and mass attenuation coefficient were also required. These coefficients were obtained from measurement and XCOM database [9, 10], and compared with the ICRU 44 protocol (soft tissue) which was previously reported by Berger and Hubbell, indicating that polyester resin is equivalent to the soft tissue of human thyroid. The phantom comprises the spinal cord, trachea and the esophagus, all based on the images of magnetic resonance imaging (MRI) as well as the dimensions given by Bouchet et al. [11]. The phantom can be used in the calibration of gamma camera for in-vivo measurements in order to determine patient-specific therapeutic activity. It can be further used to control the quality of gamma spectrometry system utilized in nuclear medicine [8].

\section{Material and Methods}

\section{Methods}

\section{Mass Attenuation Coefficient}

Mass attenuation coefficient of epoxy resin, plexiglass and soft tissue were derived from $\mathrm{XCOM}$ database in the range $0.1 \mathrm{keV}$ to 20 $\mathrm{MeV}$. Using dosimetry measurement, mass attenuation coefficient was obtained for the above-mentioned materials at $364 \mathrm{keV}$ energy (for radioactive ${ }^{131} \mathrm{I}$ source). Mass attenuation coefficient was governed by equation 1 as follows:

$$
\mu \mathrm{m}=(1 / \rho \mathrm{t}) \ln \left(\mathrm{I}_{0} / \mathrm{I}\right)
$$

Where, $\rho, \mathrm{t}, \mathrm{I}_{0}$, I and $\mu \mathrm{m}$ are absorber density, absorber thickness, initial intensity, total transmitted intensity and mass attenuation coefficient for a specific photon energy, respectively.

\section{Dosimetry}

As shown in Figure 1, a specific arrangement including collimators, ${ }^{131} \mathrm{I}$ radioactive source, samples and the dosimeter was set up for practical dosimetry, and the absorbed dose was measured by X5Cplus dosimeter. This chamber has a $0.25 \mathrm{~cm}^{3}$ sensitive volume, and its useful energy is from $40 \mathrm{keV}$ to $1.3 \mathrm{MeV}$ for $\mathrm{X}$ and gamma rays. The initial collimator was set up by 4 lead blocks with $12 \mathrm{~cm}$ thickness. The epoxy resin sample with $10 \mathrm{~mm}$ thickness and $10 \mathrm{~cm}$ diameter was fixed at $26 \mathrm{~cm}$ from the source. The second collimator was also 4 lead blocks with the same dimensions starting at $33 \mathrm{~cm}$ from the source. The detector was located at $53 \mathrm{~cm}$ from the source at the end of lead blocks. Using measured data and equations 1 and 2, mass attenuation coefficient was calculated. Photon beams were radiated from the ${ }^{131} \mathrm{I}$ source with $1.5 \mathrm{~cm}$ radius. To eliminate beta particles, a $1 \mathrm{~cm}$ thickness sheet was used.

\section{Design}

The thyroid-neck phantom was designed by CATIA V5R16 software based on geometry dimensions recommended by Bouchet et al. [11]; this phantom is a cylinder with 


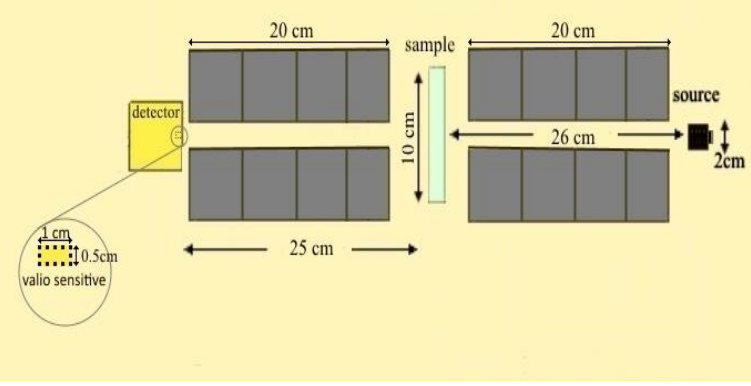

Figure 1: The radiation transport set up for practical dosimetry

$12 \mathrm{~cm}$ diameter containing a $20 \mathrm{~cm}^{3}$ thyroid. The morphology of thyroid tissue, vertebral column, trachea and esophagus were derived from MRI and CT images in the atlas of human anatomy (Figure 2)[12]. The geometry of each tissue was fed into the CATIA V5R16 software based on the above-mentioned references. The designed thyroid gland is illustrated in Figure 3.

\section{Materials}

\section{NIST XCOM}

NIST XCOM version 3.1 released by the National Institute of Standards and Technology, Maryland, USA, was used to obtain at-

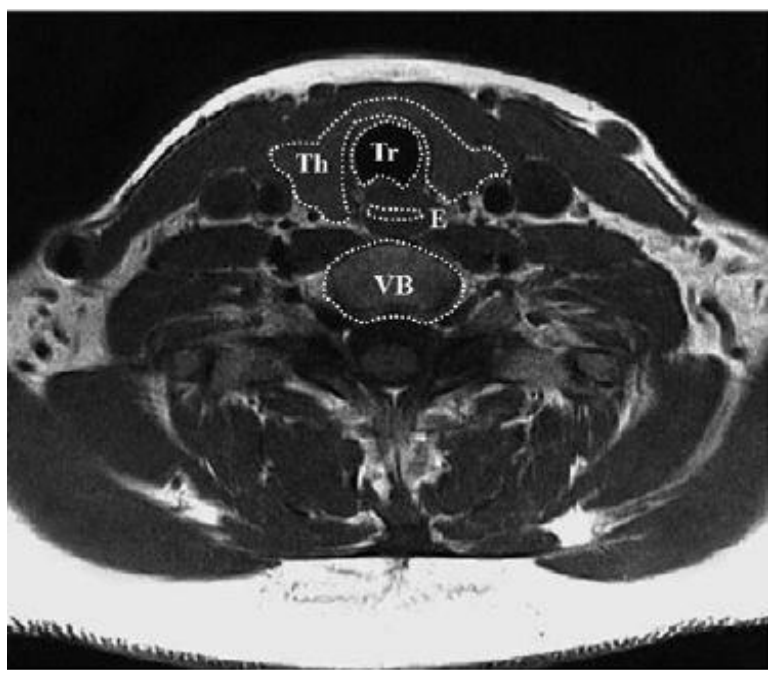

Figure 2: Human neck cross-section at C6 vertebrae indicating the thyroid gland (Th), the trachea ( $\mathrm{Tr}$ ), the esophagus (E) and the vertebral column (VB) derived from [12]

tenuation coefficients. It is a database used to calculate photon cross-section, photoelectric absorption, Compton scattering, pair production and total attenuation coefficients for elements and compounds in the range of $1 \mathrm{keV}$ to $1000 \mathrm{GeV}$. Cross-sections below and above absorption edges are accurately represented in this database [9, 10]. Mass attenuation coefficients of all materials were calculated in the

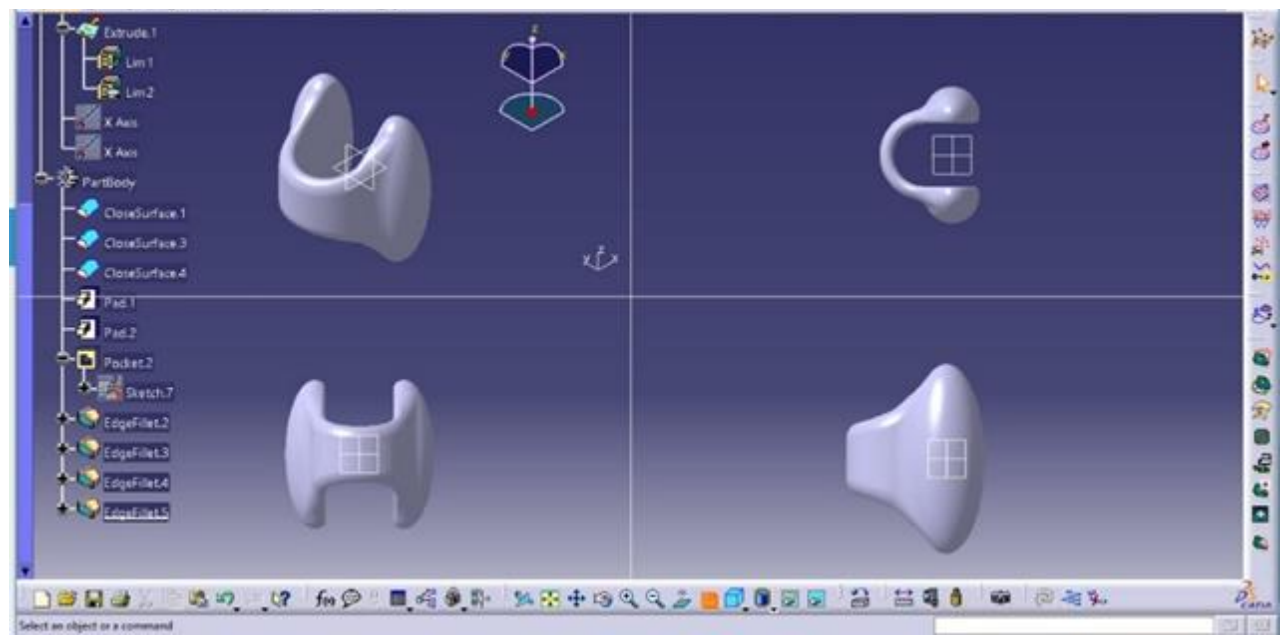

Figure 3: The thyroid gland sketch designed by CATIA V5R16 based on actual dimensions derived from the atlas of human anatomy 
range of $0.001-20 \mathrm{MeV}$ using this software.

\section{ProNest}

The sketch of neck phantom was performed by ProNest software to cut the plexiglass sheets (Figure 4). ProNest is one of the best cutting and nesting software tools in the market which was used in the $\mathrm{CNC}$ laser cutting machine.

\section{Plexyglass and Epoxy Resin}

Six plexiglass sheets, each with $2 \mathrm{~cm}$ thickness, were cut by ROTEC laser cutting machine. Since chloroform melts the outer layer of plexiglass, it was used to attach PMMA sheets to prevent air bubbles and probable inhomogeneity between the sheets.

Epoxy resin was applied to produce the thyroid phantom. This material was composed of Epichlorohydrin and Bisphenol A. This compound is electrically and chemically (against acids, bases, lipids, etc.) resistant. It is, likewise, waterproof and has little shrinkage com-

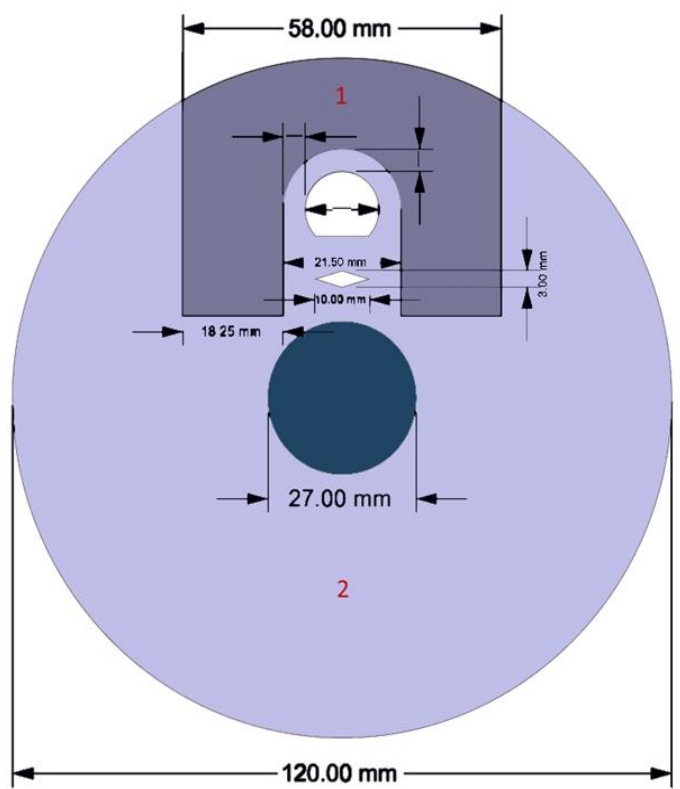

Figure 4: The thyroid-neck phantom designed by ProNest software. The central cylinder is an aluminum rod equivalent to vertebral column. The bigger cylinder was produced by PMMA and the dark section on the top is thyroid container made by epoxy resin pared to Polyester resin. (Figure 5)

The curing process which leads to solidifying the liquid epoxy resin was performed by an Amine hardener based on [13] and [14]. The epoxy: hardener ratio was 10: 5 based on the recommendations of its producing company. A homogeneous blend was solidified after 4 hours in the mold.

\section{Results and Discussion}

The comparison of the measured (dosimetry) versus calculated (XCOM) mass attenuation coefficients for soft tissue (based on ICRU 44), plexiglass and epoxy resin is illustrated

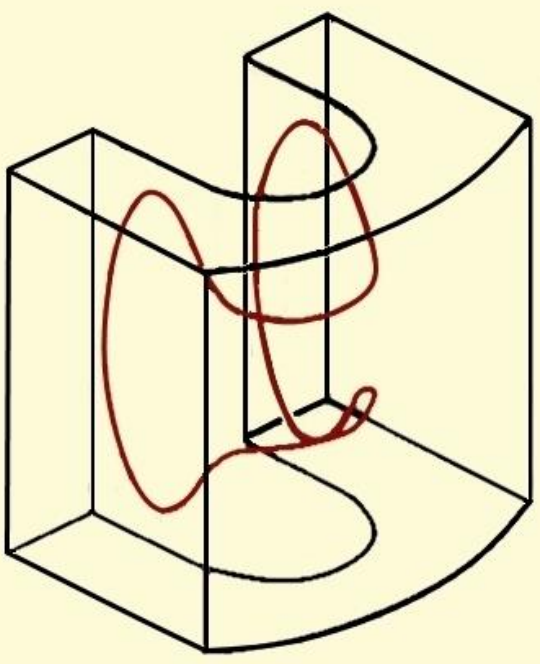

Figure 5: A section of phantom which was produced by epoxy resin material. The red line shows the location and dimension of the thyroid phantom

in Figure 6. This figure demonstrates the relative standard deviation (\%RSD) below $4 \%$ in the range of $0.05-20 \mathrm{MeV}$ between mass attenuation coefficients of the above-mentioned materials. Thus, epoxy resin can be a feasible and reliable substitution for plexiglass in the production of nuclear medicine phantoms. Due to lower effective atomic number of epoxy resin compared to PMMA and soft tissue in the range of $0.001-0.05 \mathrm{MeV}$, the $\% \mathrm{RSD}$ correlates inversely with photon energy from 


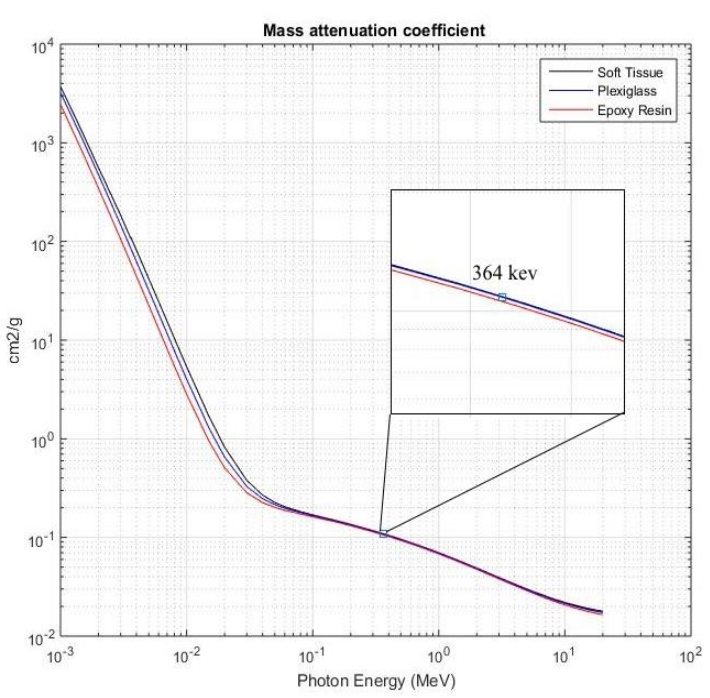

Figure 6: Mass attenuation coefficient of plexiglass, epoxy resin and soft tissue derived from XCOM database in the range 0.001 to $20 \mathrm{MeV}$

$7 \%$ to $4 \%$.

Table 1 presents the mass attenuation coefficients of soft tissue, PMMA and epoxy resin at $364 \mathrm{keV}$ energy ( ${ }^{131} \mathrm{I}$ source most probable energy). The \%RSD between PMMA and epoxy resin results at this energy is $2 \%$ which proves the XCOM outputs.

Figure 7 demonstrates the constructed phantom with a $2.7 \mathrm{~cm}$ diameter aluminum cylinder at the center and two rhombic air columns with 1 and $10 \mathrm{~mm}$ diameters as trachea. The esophagus is designed as a cylinder with 1.3 $\mathrm{cm}$ diameter. The thyroid phantom was produced as a separate section for convenience. In addition, our design allows the use of different thyroid morphologies in order to assess some pathological thyroid diseases (e.g. hyperthyroidism) in which the size and volume of the gland would vary.

Figure 8 shows a scintigraphy of the new thyroid-neck phantom captured by Infinia Hawkeye 4 gamma camera to evaluate the phantom morphology. This image proves the similarity of the new thyroid phantom and the human thyroid gland.

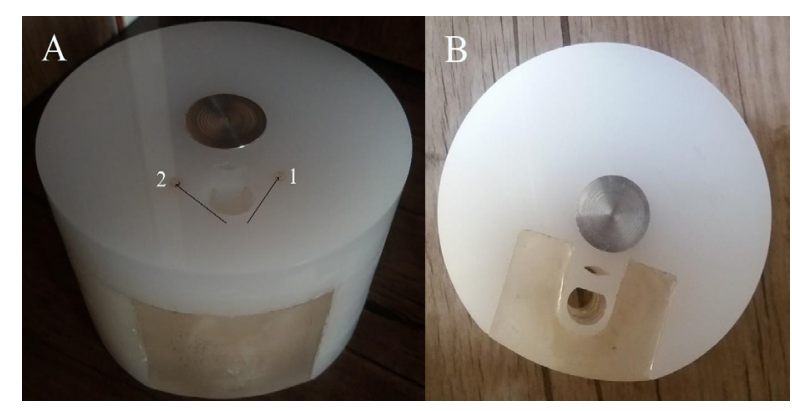

Figure 7: The constructed thyroid-neck composed of plexiglass and epoxy resin including the aluminum cylinder as vertebral column. The cavities 1 and 2 are considered for the injection into the thyroid phantom

\section{Conclusion}

This research proves the feasibility and reliability of epoxy resin compound in the production of different phantoms concurrently with poly(methyl methacrylate) in the energy range of $50 \mathrm{keV}$ to $20 \mathrm{MeV}$. The new twopiece thyroid-neck phantom reported here can be applied in the calibration of gamma camera systems, dosimetry and gamma spectrometry in the nuclear medicine field.

Table 1: Measured (dosimetry) versus calculated (XCOM) mass attenuation coefficients and relative standard deviation (\%RSD) between epoxy resin, plexiglass and soft tissue.

\begin{tabular}{cccccc}
\multirow{2}{*}{ Method } & \multicolumn{2}{c}{ Mass Attenuation Coefficient $\left(\mathbf{c m}^{2} / \mathbf{g}\right)$} & \multicolumn{2}{c}{$\%$ RSD } \\
\cline { 2 - 6 } & Plexiglass & Epoxy Resin & Soft Tissue & ER $^{*}$ PMMA & ER / Soft Tissue \\
\hline XCOM & $1.070 \mathrm{E}-01$ & $1.043 \mathrm{E}-01$ & $1.09 \mathrm{E}-01$ & $2.5 \%$ & $4.4 \%$ \\
\hline Measurement & $1.084 \mathrm{E}-01$ & $1.062 \mathrm{E}-01$ & - & $2 \%$ & -
\end{tabular}

${ }^{*} \mathrm{ER}=$ Epoxy Resin 


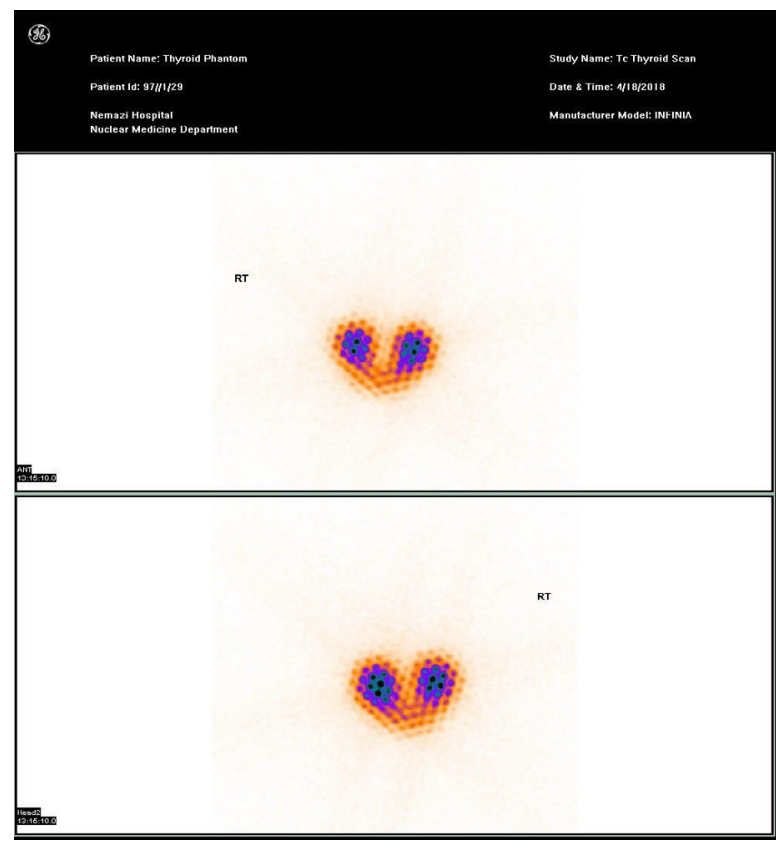

Figure 8: The scintigraphy of the new thyroid phantom captured by Infinia Hawkeye 4 gamma camera

\section{Acknowledgment}

Authors would like to thank the Vice-chancellery for Research and Technology Affairs of Shiraz University of Medical Sciences (SUMS) for supporting this research.

\section{Conflict of Interest}

There is not any relationship that might lead to a conflict of interest.

\section{References}

1. Agency IAE. Direct methods for measuring radionuclides in the human body. Wien: International Atomic Energy Agency Vienna; 1996.

2. Dantas BM, Lucena EA, Dantas AL, Araujo F, RebeIo AM, Teran M, et al. A protocol for the calibration of gamma cameras to estimate internal contamination in emergency situations. Radiat Prot Dosimetry. 2007;127:253-7. doi: 10.1093/rpd/ncm278. PubMed PMID: 17562649.

3. Siegel JA, Thomas SR, Stubbs JB, Stabin MG, Hays MT, Koral KF, et al. MIRD pamphlet no. 16: Techniques for quantitative radiopharmaceutical biodistribution data acquisition and analysis for use in human radiation dose estimates. $J$ Nucl Med. 1999;40:37S-61S. PubMed PMID: 10025848.

4. Hanscheid H, Canzi C, Eschner W, Flux G, Lus- ter M, Strigari L, et al. EANM Dosimetry Committee series on standard operational procedures for pre-therapeutic dosimetry II. Dosimetry prior to radioiodine therapy of benign thyroid diseases. Eur J Nucl Med Mol Imaging. 2013;40:1126-34. doi: 10.1007/s00259-013-2387-x. PubMed PMID: 23576099.

5. Rezaei H, Mostaghimi H, Mehdizadeh AR. Modification of Source Strength in Low-Dose-Rate Lung Brachytherapy with (125)I and (103)Pd Seeds. J Biomed Phys Eng. 2017;7:191-204. PubMed PMID: 29082211 ; PubMed Central PMCID: PMC5654126.

6. Mostaghimi H, Mehdizadeh AR, Darvish L, Akbari $\mathrm{S}$, Rezaei H. Mathematical formulation of (125)I seed dosimetry parameters and heterogeneity correction in lung permanent implant brachytherapy. $J$ Cancer Res Ther. 2017;13:436-41. doi: 10.4103/ jcrt.JCRT_978_16. PubMed PMID: 28862205.

7. Rezaei H, Zabihzadeh M, Ghorbani M, Goli Ahmadabad F, Mostaghimi $H$. Evaluation of dose enhancement in presence of gold nanoparticles in eye brachytherapy by (103)Pd source. Australas Phys Eng Sci Med. 2017;40:545-53. doi: 10.1007/ s13246-017-0555-1. PubMed PMID: 28509080.

8. Hermosilla A, Diaz Londono G, Garcia M, Ruiz F, Andrade P, Perez A. Design and manufacturing of anthropomorphic thyroid-neck phantom for use in nuclear medicine centres in Chile. Radiat Prot Dosimetry. 2014;162:508-14. doi: 10.1093/rpd/ ncu022. PubMed PMID: 24567500.

9. Berger MJ, Hubbell J. XCOM: Photon cross sections on a personal computer. National Bureau of Standards, Washington, DC (USA) Washington: National Bureau of Standards: Center for Radiation Research; 1987.

10. Berger M, Hubbell J, Seltzer S, Chang J, Coursey J, Sukumar R, et al. XCOM: Photon cross sections database, 2010. Available from: URL http://www nist gov/pml/data/xcom.

11. Bouchet LG, Bolch WE, Weber DA, Atkins HL, Poston JW, Sr. MIRD Pamphlet No. 15: Radionuclide $S$ values in a revised dosimetric model of the adult head and brain. Medical Internal Radiation Dose. J Nucl Med. 1999;40:62S-101S. PubMed PMID: 10086719.

12. Govoni AF. Pocket atlas of sectional anatomy. Computed tomography and magnetic resonance imaging. Elsevier; 2008.

13. Zhang J, Xu Y, Huang P. Effect of cure cycle on curing process and hardness for epoxy resin. Express Polymer Letters. 2009;3:534-41.

14. Zhang J, Xu Y, Huang P. Effect of cure cycle on temperature/degree of cure field and hardness for epoxy resin. e-Polymers. 2010;10. 